\title{
Acute anabolic response to $\beta$-hydroxy- $\beta$ - methylbutyrate (HMB)-free acid supplementation following heavy resistance exercise
}

\author{
JR Townsend*, JR Stout, JR Hoffman, AM Gonzalez, AR Jajtner, AJ Wells, CH Boone, KS Beyer, GT Mangine, \\ EH Robinson, GJ Pruna, JD Bohner, MS Fragala, DH Fukuda \\ From The Eleventh International Society of Sports Nutrition (ISSN) Conference and Expo \\ Clearwater Beach, FL, USA. 20-21 June 2014
}

\section{Background}

$\beta$-hydroxy- $\beta$-methylbutyrate (HMB), a metabolite of the amino acid leucine, has been shown to promote strength and lean muscle mass when supplemented in conjunction with resistance training. Recently, a new free-acid form of HMB has been shown to reach higher plasma concentrations in a shorter amount of time compared to the calcium-salt form. This higher bioavailability may rationalize acute supplementation with HMB-FA as a means to enhance the anabolic response resulting from heavy resistance training. The purpose of this study was to examine the effect of acute $\beta$-hydroxy- $\beta$-methylbutyrate-free acid (HMB-FA) on circulating concentrations of anabolic hormones following a heavy resistance exercise protocol.

\section{Methods}

Twenty resistance-trained men $(22.8 \pm 2.5$ yrs, $177.6 \pm$ $6.6 \mathrm{~cm}, 83.4 \pm 9.8 \mathrm{~kg}$ ) volunteered to participate in this study and were randomized into two groups [HMB-FA and placebo $(\mathrm{PL})]$ and performed an acute, heavy resistance exercise protocol (four sets of up to 10 repetitions of the squat, dead lift, and split squat exercises). Supplementation included $1 \mathrm{~g}$ of HMB-FA or PL consumed $30 \mathrm{~min}$ prior to exercise. Blood was sampled before (PRE), immediately post (IP), and $30 \mathrm{~min}$ post-exercise (30P). Circulating levels of testosterone (TEST), growth hormone (GH) and Insulin (INS) were assayed. A $2 \times 3$ repeated measures ANOVA was used to analyze the data. Consent to publish the results was obtained from all participants.

\section{Results}

The resistance exercise protocol produced a significant time effect for an elevation in TEST $(\mathrm{p}<0.01), \mathrm{GH}(\mathrm{p}<0.01)$ and INS $(\mathrm{p}=0.05)$ at IP with GH $(\mathrm{p}<0.01)$ and INS $(p<0.01)$ remaining elevated at 30P. A group by time interaction was observed $(\mathrm{p}=0.05)$ with plasma $\mathrm{GH}$ elevated in HMB-FA compared to PRE values $(\mathrm{p}<0.01)$ at IP and 30P. There were no differences at any other time point with TEST or INS concentrations.

\section{Conclusion}

These data indicate that HMB-FA supplementation may augment and prolong the growth hormone elevation associated with heavy resistance exercise.

\section{Acknowledgement}

This study was supported by Metabolic Technologies Inc.

Published: 1 December 2014

doi:10.1186/1550-2783-11-S1-P16

Cite this article as: Townsend et al:: Acute anabolic response to $\beta$ hydroxy- $\beta$-methylbutyrate (HMB)-free acid supplementation following heavy resistance exercise. Journal of the International Society of Sports Nutrition 2014 11(Suppl 1):P16.

\footnotetext{
* Correspondence: Jeremy.Townsend@ucf.edu
} University of Central Florida, Orlando, FL, USA 